\title{
Observation of Chain Structure of Superconducting Vortices by Lorentz Microscopy
}

\author{
${ }^{1,2,6}$ T. Matsuda, ${ }^{1}$ O. Kamimura, ${ }^{1,2,6}$ H. Kasai, ${ }^{1,2,6}$ K. Harada, ${ }^{1,6}$ T. Yoshida, ${ }^{3,6}$ T. Akashi, ${ }^{1,2,6}$ A. Tonomura, \\ ${ }^{4,6}$ Y. Nakayama, ${ }^{4,6}$ J. Shimoyama, ${ }^{4,6}$ K. Kishio, ${ }^{5,6}$ T. Hanaguri and ${ }^{5,6}$ K. Kitazawa \\ 1) Advanced Research Laboratory, Hitachi, Ltd., Hatoyama, Saitama 350-0395, Japan \\ 2) FRS, The Institute of Physical and Chemical Research (RIKEN), Wako, Saitama 351-0198, Japan \\ 3) Hitachi Instruments Service Co., Ltd., 4-28-8 Yotsuya, Shinjuku-ku, Tokyo 160-0004, Japan \\ 4) Department of Applied Chemistry, University of Tokyo, Tokyo 113-8656, Japan \\ 5) Department of Advanced Materials Science, University of Tokyo, Tokyo 113-0033, Japan \\ 6) SORST, Japan Science and Technology Corporation (JST), Nihonbashi, Chuo-ku, Tokyo 103-0027, Japan
}

Superconducting vortices (quantized magnetic flux-lines) form a triangular lattice structure inside superconductors when a magnetic field is applied. In high-temperature superconductors, however, vortices have been observed to take on an anomalous structure, called 'chain structure', when the applied magnetic field direction is tilted away from the crystallographic c-axis [1, 2]. For example, $\mathrm{Bi}_{2} \mathrm{Sr}_{2} \mathrm{CaCu}_{2} \mathrm{O}_{8+\delta}(\mathrm{Bi}-2212)$ vortices alternately form liner rows called 'vortex chains' and normal triangular domains [1]. As this chain structure has never been observed in metallic superconductors, the structure is considered to reflect the highly anisotropic layered structure of the materials in which they do appear [3]. Here we report the first observation of the chain structure of Bi-2212 vortices [4] and their unexpected dynamical behaviors by Lorentz microscopy using 1-MV field emission electron microscope [5].

Bi-2212 thin films of 400-nm thick were prepared by cleaving from a single crystal which was made of ordinary floating zone method, and mounted on the cooling holder at $30^{\circ}$ tilted to the electron beam (z-axis) and around the x-axis. The specimen was inserted into the microscope column that had the direction-free magnetic field application system described elsewhere [6]. Figure 1 shows the experimental setup and how we applied the magnetic field; it also indicates our experimental coordinates. A magnetic field of 1 - $10 \mathrm{mT}$ was applied to, the film at $70-90^{\circ}$ from the z-axis. The azimuth angle of the magnetic field was selected freely us on experimental condition. The azimuth is along the X-axis in Fig. 1, and the chain structure was observed along the same direction.

Figure 2 shows a typical Lorentz micrograph of the chain structure, which is highlighted by white arrows, at temperature of $50 \mathrm{~K}$. As seen in the figure, the spacing of the chain vortices is narrower than, and therefore incommensurate with that of the surrounding triangular-lattice vortices. When we applied a glancing magnetic field to the specimen (y-direction in Fig. 1), the vortices on the chain rows were connected and took on linear form in the image because of their geometrical configuration.

We found some surprising behaviors of the vortices on the chains. All of the vortices on the chains were clearly observed at low temperature in field-cooled and/or at a low magnetic field the azimuth of which was along the x-direction. Some of vortices on the chain, however, disappeared at higher temperature and higher magnetic field. An example at $57 \mathrm{~K}$ is shown in Fig. 3. The contrasts of the vortices (a) and (b) in Fig. 3 are high, but the contrast of other vortices along the chain show gradual decreases compared to the vortices (a) and (b). Such partial disappearances were extended with increasing temperature. 
We consider that the reason for the vortex-image disappearance results from the oscillation of vortices along the chain direction being too fast to be observed even by using the TV-rate observation. This can be explained by the fact that vortices (a) and (b) are located at locally commensurate positions with the surrounding triangular vortex-lattice, i.e. each vortex (a) or (b) is firmly fixed by the four surrounding vortices and behaves like a node of the string oscillation. Other vortices between the nodes are not in stable positions and can move easily.

This local oscillation of vortices may be too simple to explain the new findings of vortices disappearance. More detailed experiments and theoretical simulations are now in progress in order to determine the characteristics of the vortices.

\section{References}

[1] C. A. Bolle, et al., Phys. Rev. Lett. 66 (1991) 112.

[2] P. L. Gammel et al., Phys. Rev. Lett. 68 (1992) 3343.

[3] A. E. Koshelev, Phys. Rev. Lett. 83 (1999) 187.

[4] T. Matsuda et al., Science 294 (2001) 2136.

[5] T. Kawasaki et al., Appl. Phys. Lett. 76 (2000) 1342.

[6] K. Harada et al., in this proceedings.

[7] We areverygrateful to Dr. F. Nori, AKoshelev, T. Hashizume, T. On,ogi

P. L. Gammel and D. J. Bishøpr theiruseful suggestion

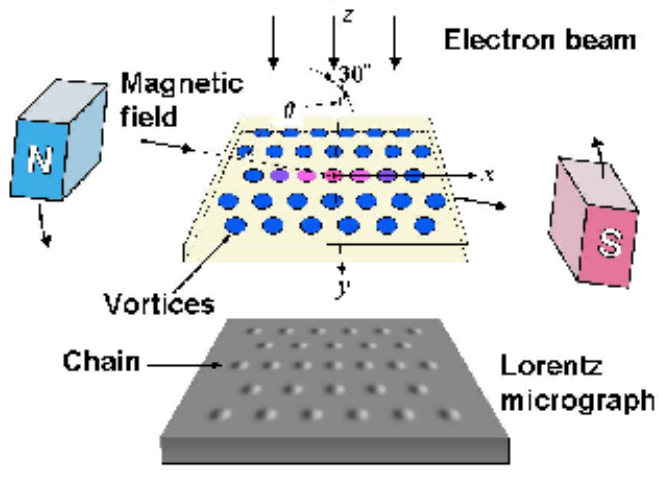

Figure 1 Schematic of experimental setup. A parallel electron beam was incident along the z-axis and film was tilted by $30^{\circ}$ around the y-axis.

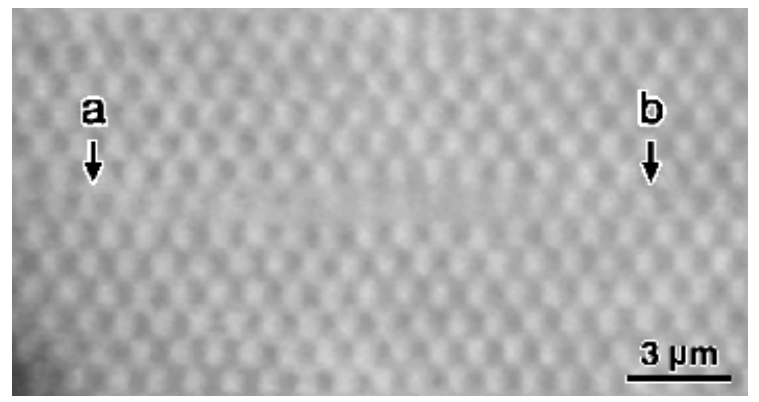

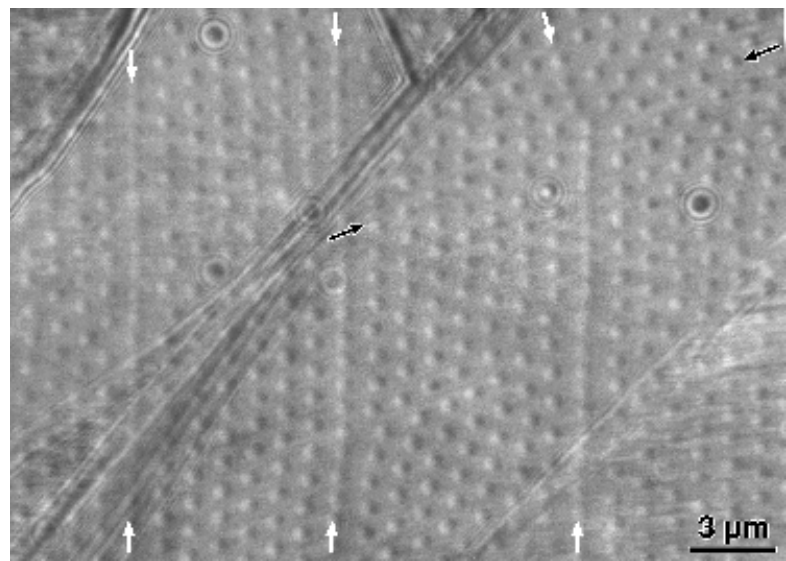

Figure 2 Lorentz micrograph of chain-lattice structure of vortices in Bi-2212 film 400-nm thick at $50 \mathrm{~K}$, with $1 \mathrm{mT}$ and tilt of $80^{\circ}$. Three chains of vortices in vertical direction are indicated by white arrows.

Figure 3 Example of clearly disappearing vortex-images in single chain at $57 \mathrm{~K}, 5 \mathrm{mT}$ and $80^{\circ}$ 